\title{
Letters to the Editor-August 26, 2020
}

\author{
Physicists returning to the lab after the long shutdown describe family \\ science chats, paperwork headaches, and raising the next generation of \\ experimentalists.
}

7 his is our final series of letters about how physicists are finding the return to the lab during the pandemic. Thank you to all who shared their experiences.

\section{The Quest Must Go On}

"Mommy, try this Zoom background," my 9-year-old says. I'm now embedded in an STM-imaged gold nanocrystal! A trick he picked up making videos to entertain his cousins.

As a quantum condensed matter theorist on sabbatical in San Diego this past academic year, I've become accustomed to working remotely with my students in Illinois and my collaborators worldwide. Throughout the pandemic challenges, we light up while discussing entanglement growth in topological systems or hollow condensates aboard the International Space Station. The situation and science strengthen parental bonds too: I'm making progress on a popular book on quantum physics and relativity that I began with my late father, a black-hole physicist. Phone calls with my

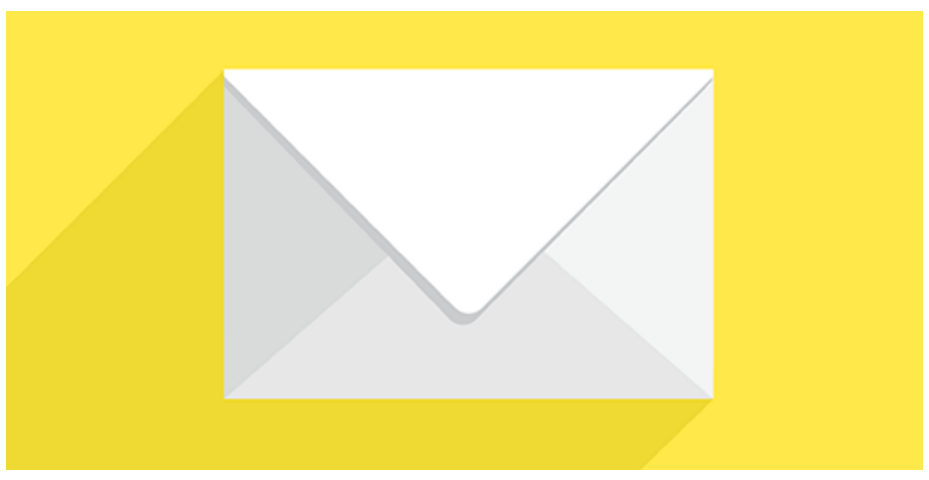

Credit: APS/Carin Cain biophysicist mother, isolated during India's lockdown, have turned into COVID-19 spike-protein research.

What's next? Still no in-person meetings nor equations scribbled over coffee chats. Experimental labs remain semi-closed. Our universities now seek to reopen-to drastically new norms.

On uplifting beach walks, I contemplate the gravitational waves that wash over us from distant black holes, the quantum stardust soup we are made of, and the miracle of life and human connections. For our community and youngsters, our scientific quest must go on-safely, compassionately.

- Smitha Vishveshwara is a Professor of Physics at the University of Illinois at Urbana-Champaign and was a Margaret Burbidge Visiting Professor in the 2019-2020 academic year at the University of California, San Diego.

\section{Paperwork is Dangerous}

As Chair of one of the two physics departments at my institute, the most challenging and stressful part of working from home has been dealing with bureaucratic red tape. As an example, we recently spent a lot of time deciding how a retired professor should sign a contract and how to shuttle this contract from my department office to an office downstairs. Should I "summon" an administrative assistant-who is now working remotely-to come with the retired professor to the institute with the sole purpose of printing out a form, signing it, and transporting it to another office, where another assistant would be waiting?

This task wastes everyone's time and exposes us all to unnecessary risks. We ultimately found a solution, but it still 


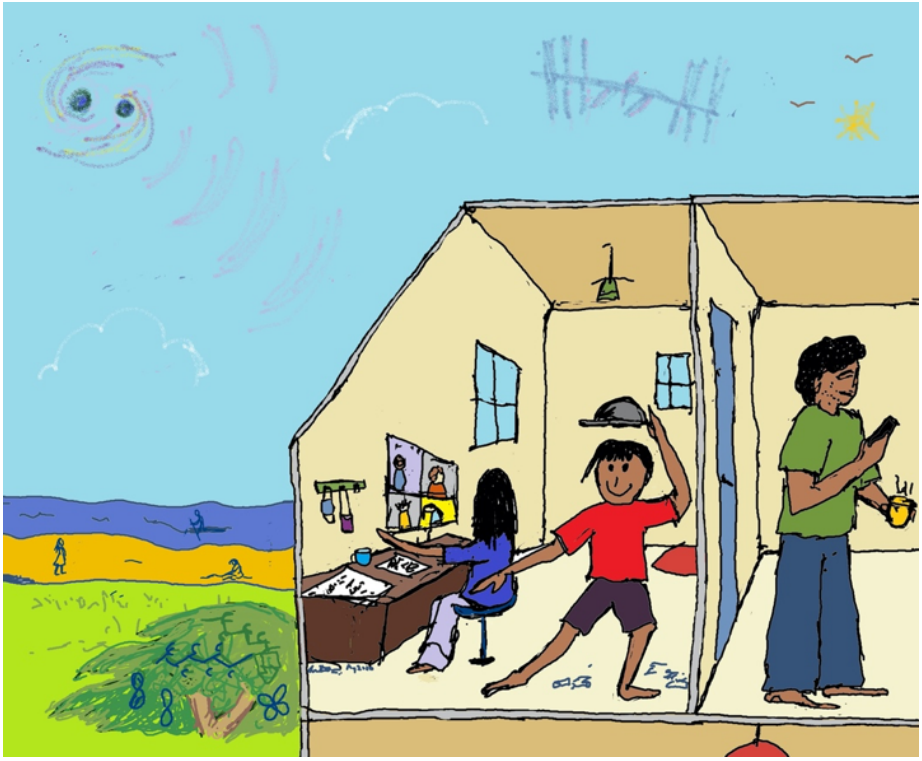

Sabbatical in San Diego during the pandemic.

Credit: S. Vishveshwara/U. Illinois Urbana-Champaign

required some people to meet in person. Why not just use electronic signatures and avoid risks altogether? (One positive of the pandemic is that we have highlighted many inefficiencies in what the institute does.)

As my institute discusses partially reopening labs, key cards to enter our buildings pose another challenge. Currently an administrative assistant has to hand deliver the cards to whoever requests one, even though our card readers also accept typed-in passwords. We still have a long way to go toward accepting that our current condition isn't temporary and that we have to adapt.

- Carlos Egues is a professor at the São Carlos Institute of Physics at the University of São Paulo, Brazil.

\section{Commitment to Training New Students}

In early March, Los Alamos National Laboratory (LANL) established that telecommuting would be the new default. My husband, who is also a co-worker, and I adapted easily and focused on writing manuscripts and grant proposals and on planning for the eventual reopening.

New Mexico's governor took aggressive measures that allowed

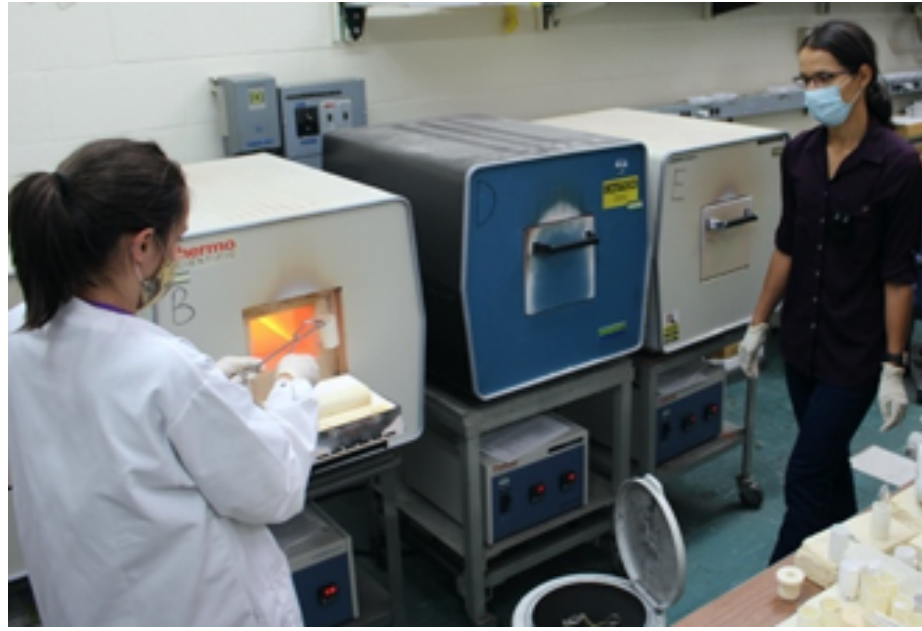

Master's student Shannon Fender (left) removes samples from the furnace under the supervision of staff scientist Priscila Rosa (right). Credit: S. Thomas/LANL

the state to start reopening in late May. LANL began a new routine of limited operations with onsite activities restricted to $50 \%$ of normal. Employees must wear masks, keep six feet apart, and disinfect common surfaces. As an experimental physicist, it's intuitive to put on a mask and gloves. It is also straightforward to treat surfaces as if they are contaminated-just imagine they are covered with radioactive material! Nevertheless, social distancing presents a challenge, as science is inevitably social. When I see a colleague in the hallway or the lab, my first instinct is to approach and ask how things are going. We've had to fundamentally re-train ourselves.

This environment is particularly hard on students and postdocs, who have limited time to complete their projects. Shannon Fender, a master's student from the University of Oregon, joined our group at the end of January for a one-year appointment. After her training, she had a month before labwork stopped. Now we are trying to make the most of her remaining time. Teaching lab skills to a new student is especially challenging under social distancing requirements. Nonetheless, it is key that we continue to train and support junior scientists to ensure a strong and diverse future for our scientific community.

- Priscila Rosa is a Staff Scientist in the Materials Physics and Applications Division at Los Alamos National Laboratory, New Mexico. 


\section{Achieving Milestones Amid COVID-19}

The beginning of 2020 saw years of plans converging for the ITER Organization (the international collaboration that works toward demonstrating the scientific and technological feasibility of fusion energy for peaceful purposes). Construction has reached completion on major components for the thermonuclear fusion reactor. Complex delivery protocols were set in motion to bring these large pieces onsite in Cadarache, France. Unfortunately, the start of COVID-19 necessitated some major changes to starting assembly.

ITER quickly enacted safety measures for on-site essential staff, prioritizing worker welfare while doing its best to stay on track. The new work-from-home situation for non-essential staff has had minimal impact on most of my division. A lot of our work lies in the realm of simulations. While adjusting to the work-from-home lifestyle, frequent boosts in morale came from the daily ITER Bulletin that keeps us all up to date on progress. Workers here are still striving to make fusion energy a reality.
By mid-May, France began to lift restrictions and non-essential workers started voluntarily returning to the work site. Bus rides to the site operate at half capacity with staggered seating, our beloved café is closed for everything except lunch, all meetings are virtual, and masks are required. In the end, we all came together online for a virtual "start of assembly" celebration. Sharing that moment was fantastic, even at a distance. With the cryostat base safely installed in the tokamak pit, work is moving forward. New components are constantly arriving from across the world. We're now testing a working schedule of two days on-site, three days off-site, and are learning as we go while maintaining both progress and safety.

- Jonathan Coburn is a Monaco Postdoctoral Fellow within the Science Division of the ITER Organization, Cadarache, France. 